\title{
Risk Factors for Pneumonia in Children under 5 Years in a Teaching Hospital in Nepal \\ Karki S, ${ }^{1}$ Fitzpatrick AL, ${ }^{2}$ Shrestha ${ }^{3}$
}

${ }^{1}$ Department of Nursing

Kathmandu University School of Medical Sciences, Dhulikhel, Nepal.

${ }^{2}$ Department of Family Medicine

Epidemiology and Global Health, University of Washington, Seattle, WA USA

${ }^{3}$ Department of Nursing

Tribhuwan University, Institute of Medicine, Kathmandu, Nepal.

\section{Corresponding Author}

Sita Karki

Department of Nursing

Kathmandu University School of Medical Sciences, Dhulikhel, Nepal.

Email: sitakarki_2000@yahoo.com

\section{Citation}

Karki S, Fitzpatrick AL, Shrestha S. Risk Factors for Pneumonia in Children under 5 Years in a Teaching Hospital in Nepal. Kathmandu Univ Med J 2014;48(4):247-52.

\section{ABSTRACT}

\section{Background}

Pneumonia is a leading cause of mortality among children under-five years of age globally. The WHO (2014) has reported that indoor air pollution caused by cooking and heating with biomass fuel, living in crowed homes and parenting smoking are risk factors of pneumonia.

\section{Objectives}

The objective of the study was to identify the risk factors for pneumonia among children under- 5 years of age.

\section{Methods}

A case control study was carried out among the mothers having under- 5 years children who were admitted in the paediatric ward of Dhulikhel Hospital in 2012/13. A convenience sampling technique was used to select 50 children with pneumonia and 150 children with non-pneumonia diseases matched on age, sex and setting. A semi-structured interview consisting of questions related to risk factors for pneumonia was used to collect data from mothers of both cases and controls.

\section{Results}

Sex of the child did not differ by case/control group whereas the children with pneumonia were slightly older with $26 \%$ cases and $15 \%$ controls older than 3 years of age. Mother's education was similar in both groups as was family income. Living in a household with a chulo with smoke increased the odds of having pneumonia significantly, with the risk almost 4 times greater if the chulo was located within the same building (OR: $3.76,95 \% \mathrm{Cl}: 1.20-11.82, \mathrm{p}=0.02$ ). Children who had diarrhea in the past 3 months were protected from pneumonia (OR: $0.38,95 \% \mathrm{Cl}: 0.18$ $0.82, p=0.01)$. An increasing trend of pneumonia was observed among children of tobacco smoking parents with greater risk if both parents smoked; it was, however, not statically significant (OR: $2.21,95 \% \mathrm{Cl}: 0.56-8.82, \mathrm{p}=0.26$ ).

\section{Conclusion}

The present study suggests that two factors related to smoke, presence of a smoky chulo in a household and both parents smoking, are modifiable risk factors related to pneumonia in young children. Reliable longitudinal studies, interventions, and programs to educate parents in prevention are important for reducing mortality and morbidities related to acute respiratory illnesses in Nepal.

\section{KEY WORDS}

Mothers, pneumonia, risk factors, under 5 children. 


\section{INTRODUCTION}

Pneumonia is one of the major public health problems in children under 5 years of age. According to the World Health Organization (WHO), more than 150 million episodes of pneumonia occur every year and these account for more than $95 \%$ of all new cases worldwide. ${ }^{1}$ According to another report of WHO, nearly 2 million children under 5 years die of pneumonia each year around the world. It is estimated that 500 to 900 million acute respiratory infection (ARI) episodes occur per year in developing countries. ${ }^{2}$ Previous research has shown that in low resource settings, risk factors for pneumonia in children have included malnutrition, inadequate paternal education, bad ventilated living room, and smoking habits of parents. ${ }^{3}$

Pneumonia is an important cause of morbidity and mortality of children especially in developing countries. The Nepal Department of Health Services reported that acute respiratory illness (ARI) is the number one killer disease of children in Nepal. ${ }^{4}$ The Ministry of Health and Population (MOHP) recognizes ARI as one of the major public health problems in Nepal among children under 5 years of age. The number of deaths due to ARI is around 28,000 children in Nepal each year. ${ }^{5}$

This study was carried out to identify the risk factors for pneumonia among children age 1 month to 5 years in Nepal.

\section{METHODS}

A hospital-based case-control study was conducted in the pediatric ward of Dhulikhel Hospital from June 2012 to May 2013 after receiving approval from the Ethical Review committee of Dhulikhel Hospital. Informed verbal consent was obtained from each respondent prior to collecting data. The subjects were assured of the confidentiality of their information and they were assured that they would be allowed to refuse to participate in the study at any time if they wished. The mothers who attended with sick children aged 1 month -5 years were considered the study population for this research.

Fifty children age 5 years and under who were admitted into the pediatric ward of Dhulikhel Hospital with pneumonia but with no under-lying chronic illnesses such as Asthma, Heart disease, and Down's Syndrome, were enrolled in the study as cases. Controls included children who were admitted to the ward during the study period with other diseases but no pneumonia and no under-lying chronic illnesses. Three controls per case were matched on age, sex, and hospital setting. Mothers of each child were identified for study participation to provide demographic and other health information needed for this study.

A semi-structured interview with the mother of each child was used for data collection. The first part of the research instrument consisted of items related to socio-demographic characteristics of the mother, the second part included child-related data and the third part included homerelated factors. The content validity of the instrument was established by seeking the opinion of pediatric consultants and a pediatric nursing teacher. The instrument was then translated into Nepali language and reviewed by a language expert for comprehensibility and simplicity of language, and for consistency of the content. The instrument was pretested on 10 women from Dhulikhel Hospital, who were excluded from the final study. The subjects were selected by using a non-probability purposive sampling technique.

The collected data were reviewed daily for completeness and accuracy. Edited data were entered into the Statistical Package for Social Science Software (SPSS) version 16.0. Data were analyzed using frequencies and percentages to describe the sample. We used multiple logistic regressions to evaluate the increased odds of having pneumonia by specific risk factors collected in the interview. Odds rations (ORs), 95\% confidence intervals (Cls) and p-values for unadjusted and adjusted models were estimated. Models evaluating child characteristics and risk of pneumonia were adjusted for child age and sex; those evaluating factors related to the mother's characteristics and home environment were adjusted for mother's age, education and family income. A p-value of $<0.05$ was considered significant in discussions of factors related to the odds of having pneumonia.

\section{RESULTS}

Result of the study are presented as descriptive statistics shown by case/control status and results of regression models. Table 1 provides maternal characteristics of children with and without pneumonia. Table 2 presents child-related characteristics, and Table 3 displays home related factors. Tables 4,5 and 6 present multivariate analyses of logistic regression models investigating associations between specific risk factors and odds of having pneumonia.

As can be seen in Table 1, The majority of mothers of cases (88.0\%) and controls (76.7\%) were young (less than 20 years) when their first child was born. In regard to education, $72 \%$ mothers of cases and $65.3 \%$ of controls had primary or secondary levels of education. The majority of the mothers of cases $(76.0 \%)$ as well as controls $(80.7 \%)$ belonged to Hindu religion; and nearly half of the respondents $(44.0 \%$ mothers of cases and $45.3 \%$ mother of controls) were from single family homes. In terms of income, about $18 \%$ respondents did not have sufficient money beyond that required for basic needs.

Table 2 shows that the majority of the case $(46.0 \%)$ and control children $(44.7 \%)$ were in the $1-3$ year age group. Pneumonia was common in male children (55.5\%) in both groups; and the majority of the children (75.0\%) were born in health institutions at a normal weight (78.4\%). Gestation 
Table 1. Socio-demographic Characteristics of Mothers of Children with and without Pneumonia

\begin{tabular}{|c|c|c|c|}
\hline Maternal Characteristic & $\begin{array}{l}\text { Pneumonia } \\
n=50 \\
\text { No. (\%) }\end{array}$ & $\begin{array}{l}\text { No Pneumonia } \\
n=150 \\
\text { No. (\%) }\end{array}$ & $\begin{array}{l}\text { Total } \\
\mathrm{n}=200)^{*} \\
\text { No. (\%) }\end{array}$ \\
\hline $\begin{array}{l}\text { Age in years } \\
\text { Less than } 20 \\
20-29 \\
30 \text { and above }\end{array}$ & $\begin{array}{l}44(88.0) \\
1(2.0) \\
5(10.0)\end{array}$ & $\begin{array}{l}115(76.7) \\
12(6.5) \\
23(15.3)\end{array}$ & $\begin{array}{c}159(79.5) \\
13(6.5) \\
28(14.0)\end{array}$ \\
\hline $\begin{array}{l}\text { Religion } \\
\text { Hindu } \\
\text { Buddhist } \\
\text { Other }\end{array}$ & $\begin{array}{l}38(76.0) \\
11(22.0) \\
1(2.0)\end{array}$ & $\begin{array}{l}121(80.7) \\
20(13.3) \\
9(6.0)\end{array}$ & $\begin{array}{c}159(79.5) \\
3115.5) \\
10(5.0)\end{array}$ \\
\hline $\begin{array}{l}\text { Education of Mother } \\
\text { None/illiterate } \\
\text { Primary } \\
\text { Secondary } \\
\text { College or more }\end{array}$ & $\begin{array}{l}6(12.0) \\
18(36.0) \\
18(36.0) \\
8(16.0)\end{array}$ & $\begin{array}{l}23(15.3) \\
42(28.0) \\
56(37.3) \\
29(19.3)\end{array}$ & $\begin{array}{l}29(14.5) \\
60(30.0) \\
74(37.0) \\
37(18.5)\end{array}$ \\
\hline $\begin{array}{l}\text { Economic status } \\
\text { Sufficient } \\
\text { Insufficient }\end{array}$ & $\begin{array}{l}44(88.0) \\
6(12.0)\end{array}$ & $\begin{array}{l}119(79.3) \\
31(20.7)\end{array}$ & $\begin{array}{c}163(81.5) \\
37(18.5)\end{array}$ \\
\hline $\begin{array}{l}\text { Type of Family } \\
\text { Single } \\
\text { Joint } \\
\text { Extended }\end{array}$ & $\begin{array}{l}22(44.0) \\
20(40.0) \\
8(16.0)\end{array}$ & $\begin{array}{l}68(45.3) \\
58(38.7) \\
24(16.0)\end{array}$ & $\begin{array}{l}90(45.0) \\
78(39.0) \\
32(16.0)\end{array}$ \\
\hline
\end{tabular}

*numbers may not sum to 200 due to missing data in some cells.

Table 3. Selected Home Environment Related Characteristics of Children with and without Pneumonia

\begin{tabular}{|c|c|c|c|}
\hline Home Characteristic & $\begin{array}{l}\text { Pneumonia } \\
n=50 \\
\text { No. (\%) }\end{array}$ & $\begin{array}{l}\text { No Pneumonia } \\
n=150 \\
\text { No. (\%) }\end{array}$ & $\begin{array}{l}\text { Total } \\
n=200)^{*} \\
\text { No. (\%) }\end{array}$ \\
\hline $\begin{array}{l}\text { Ventilation } \\
\text { Well-ventilated } \\
\text { Poorly ventilated }\end{array}$ & $\begin{array}{l}33(66.0) \\
17(34.0)\end{array}$ & $\begin{array}{l}104(69.3) \\
46(30.7)\end{array}$ & $\begin{array}{l}137(68.5) \\
63(31.5)\end{array}$ \\
\hline $\begin{array}{l}\text { Smoking Status of } \\
\text { Parents } \\
\text { Neither parent smokes } \\
\text { Father smokes } \\
\text { Mother smokes } \\
\text { Both parent smoke }\end{array}$ & $\begin{array}{l}39(78.0) \\
6(12.0) \\
1(2.0) \\
4(8.0)\end{array}$ & $\begin{array}{l}129(86.0) \\
11(7.3) \\
3(2.0) \\
7(4.7)\end{array}$ & $\begin{array}{l}168(84.0) \\
17(8.5) \\
4(2.0) \\
11(5.5)\end{array}$ \\
\hline $\begin{array}{l}\text { Condition of Chulo } \\
\text { mokeless-separate } \\
\text { building }\end{array}$ & $11(22.0)$ & $57(38.0)$ & $68(34.0)$ \\
\hline $\begin{array}{l}\text { Smokeless - same } \\
\text { building }\end{array}$ & $9(18.0)$ & $22(14.7)$ & $31(15.5)$ \\
\hline $\begin{array}{l}\text { Smoke - separate } \\
\text { building }\end{array}$ & $20(40.0)$ & $50(33.3)$ & $70(35.0)$ \\
\hline Smoke - same building & $10(20.0)$ & $21(14.0)$ & $31(15.5)$ \\
\hline
\end{tabular}

*Numbers may not sum to 200 due to missing data in some cells.

for the majority of the case $(80.0 \%)$ and control $(76.7 \%)$ children was greater than 37 weeks. About $90 \%$ of the children were fed colostrum after birth; and $72.0 \%$ of the case and $68.7 \%$ of control children were exclusively breast fed until 4-6 months. Majority of the children $(62.0 \%$ and $57.3 \%)$ were fully vaccinated in both groups. Among the children $76.0 \%$ case and $56.0 \%$ control children did not have diarrhea in the past 3 months; and more than $50 \%$ had attended a child care center in both groups.

A majority of mothers of case (66.0\%) and control (69.3\%) children lived in well ventilated houses (Table 3). Rates of parental smoking overall was low with $78.0 \%$ of case and
Table 2. Selected Characteristics of Children with and without Pneumonia

\begin{tabular}{|c|c|c|c|}
\hline Characteristic & $\begin{array}{l}\text { Pneumonia } \\
n=50 \\
\text { No. (\%) }\end{array}$ & $\begin{array}{l}\text { No Pneumonia } \\
n=150 \\
\text { No. (\%) }\end{array}$ & $\begin{array}{l}\text { Total } \\
n=200 * \\
\text { No. (\%) }\end{array}$ \\
\hline $\begin{array}{l}\text { Age Group } \\
\text { Under } 1 \text { year } \\
1-3 \text { years } \\
\text { Above } 3 y r s\end{array}$ & $\begin{array}{l}14(28.0) \\
23(46.0) \\
13(26.0)\end{array}$ & $\begin{array}{l}61(40.7) \\
67(44.7) \\
22(14.6)\end{array}$ & $\begin{array}{l}75(37.5) \\
90(45.0) \\
35(17.5)\end{array}$ \\
\hline $\begin{array}{l}\text { Sex of Child } \\
\text { Male } \\
\text { Female }\end{array}$ & $\begin{array}{l}30(60.0) \\
20(40.0)\end{array}$ & $\begin{array}{l}81(54.0) \\
69(46.0)\end{array}$ & $\begin{array}{l}111(55.5) \\
89(44.5)\end{array}$ \\
\hline $\begin{array}{l}\text { Birthplace of Child } \\
\text { Home } \\
\text { Health Institution }\end{array}$ & $\begin{array}{l}14(28.0) \\
36(72.0)\end{array}$ & $\begin{array}{l}36(24.0) \\
114(76.0)\end{array}$ & $\begin{array}{l}50(25.0) \\
150(75.0)\end{array}$ \\
\hline $\begin{array}{l}\text { Birth Weight of Child } \\
\text { Normal ( } 2.5 \mathrm{~kg} \text { or more) } \\
\text { Underweight (less than } \\
2.5 \mathrm{~kg} \text { ) }\end{array}$ & $\begin{array}{l}38(76.0) \\
12(24.0)\end{array}$ & $\begin{array}{l}111(79.3) \\
29(20.7)\end{array}$ & $\begin{array}{l}149(78.4) \\
41(21.6)\end{array}$ \\
\hline $\begin{array}{l}\text { Gestational Age of Child } \\
37 \text { weeks or less } \\
\text { Above } 37 \text { weeks }\end{array}$ & $\begin{array}{l}10(20.0) \\
40(80.0)\end{array}$ & $\begin{array}{l}35(23.3) \\
115(76.7)\end{array}$ & $\begin{array}{l}45(22.5) \\
15(77.5)\end{array}$ \\
\hline $\begin{array}{l}\text { Colostrum Feeding given } \\
\text { Yes } \\
\text { No }\end{array}$ & $\begin{array}{l}47(94.0) \\
3(6.0)\end{array}$ & $\begin{array}{l}133(88.7) \\
17(11.3)\end{array}$ & $\begin{array}{c}180(90.0) \\
20(10.0)\end{array}$ \\
\hline $\begin{array}{l}\text { Duration of Breast } \\
\text { feeding } \\
4-6 \text { month } \\
\text { Less than } 4 \text { months } \\
\text { Greater than } 6 \text { months }\end{array}$ & $\begin{array}{l}36(72.0) \\
9(18.0) \\
5(10.0)\end{array}$ & $\begin{array}{l}103(68.7) \\
33(22.0) \\
14(9.3)\end{array}$ & $\begin{array}{c}139(69.5) \\
42(21.0) \\
19(9.5)\end{array}$ \\
\hline $\begin{array}{l}\text { Immunization Status of } \\
\text { Child } \\
\text { Fully vaccinated } \\
\text { Not fully vaccinated }\end{array}$ & $\begin{array}{l}31(62.0) \\
19(38.0)\end{array}$ & $\begin{array}{l}86(57.3) \\
64(42.7)\end{array}$ & $\begin{array}{l}117(58.5) \\
83(41.5)\end{array}$ \\
\hline $\begin{array}{l}\text { Diarrhoea within past } 3 \\
\text { months } \\
\text { No } \\
\text { Yes }\end{array}$ & $\begin{array}{l}38(76.0) \\
12(24.0)\end{array}$ & $\begin{array}{l}82(56.0) \\
65(44.0)\end{array}$ & $\begin{array}{l}122(61.0) \\
78(39.0)\end{array}$ \\
\hline $\begin{array}{l}\text { Attending Child Care } \\
\text { Center } \\
\text { Yes } \\
\text { No }\end{array}$ & $\begin{array}{l}32(64.0) \\
18(36.0)\end{array}$ & $\begin{array}{l}118(78.7) \\
32(21.3)\end{array}$ & $\begin{array}{c}150(75.0) \\
50(25.0)\end{array}$ \\
\hline
\end{tabular}

$86.0 \%$ of control children's parents reported not to smoke. In terms of the chulo used in the household, case (40.0\%) and control (33.3\%) children's parents had used a smoky chulo but lived in separate houses.

In unadjusted models (Table 4), mothers who were less than 20 years and more than 30 years old were less likely to have children with pneumonia compared to mothers age 20-29 but the relationship was not significant. A borderline significant association was found showing women 30 years and older to be $63 \%$ less likely to have children with pneumonia present at the hospital $(p=0.09)$. Similarly, religion and education status of mothers and economic status of family did not have any significant effect on the pneumonia status of their children. While the children who lived in joint and extended families had an increased odds of having pneumonia than did the children living in single family households, these associations were also not significant. 
Table 4. Associations between Maternal Characteristics and Odds of Children Having Pneumonia

\begin{tabular}{|c|c|c|c|c|c|}
\hline \multirow[t]{2}{*}{$\begin{array}{l}\text { Maternal } \\
\text { Characteristics }\end{array}$} & \multirow{2}{*}{$\begin{array}{l}\text { Pneu- } \\
\text { monia } \\
\text { Yes/No }\end{array}$} & \multicolumn{2}{|l|}{ Unadjusted } & \multicolumn{2}{|c|}{$\begin{array}{l}\text { Adjusted for } \\
\text { Demographics }\end{array}$} \\
\hline & & OR $(95 \% \mathrm{Cl})$ & $\mathrm{P}$ & OR $(95 \% \mathrm{Cl})$ & $\mathrm{p}$ \\
\hline \multicolumn{6}{|l|}{$\begin{array}{l}\text { Age of Mother } \\
\text { (years) }\end{array}$} \\
\hline $20-29$ years & $44 / 115$ & 1.00(reference) & 0.21 & 1.00(reference) & 0.11 \\
\hline Under 20 years & $1 / 12$ & $0.22(0.03-1.73)$ & 0.15 & $0.23(0.03-1.89)$ & 0.18 \\
\hline 30 and older & $5 / 23$ & $0.57(0.20-1.59)$ & 0.28 & $0.37(0.12-1.14)$ & 0.09 \\
\hline \multicolumn{6}{|l|}{ Religion } \\
\hline Hindu & $38 / 121$ & 1.0(reference) & 0.23 & 1.00(reference) & 0.19 \\
\hline Buddhist & $11 / 20$ & $1.75(0.77-3.98)$ & 0.18 & $1.97(0.82-4.73)$ & 0.13 \\
\hline Other & $1 / 9$ & $0.35(0.04-2.88)$ & 0.33 & $0.38(0.04-3.32)$ & 0.38 \\
\hline \multicolumn{6}{|l|}{$\begin{array}{l}\text { Education of } \\
\text { Mother }\end{array}$} \\
\hline Secondary & $18 / 56$ & 1.0(reference) & 0.72 & 1.00 (reference) & 0.86 \\
\hline None/illiterate & $6 / 23$ & $0.81(0.29-2.30)$ & 0.70 & $1.03(0.32-3.29)$ & 0.96 \\
\hline Primary & $18 / 42$ & $1.33(0.62-2.87)$ & 0.46 & $1.37(0.60-3.14)$ & 0.45 \\
\hline $\begin{array}{l}\text { College or } \\
\text { more }\end{array}$ & $8 / 29$ & $0.86(0.33-2.21)$ & 0.75 & $0.96(0.36-2.54)$ & 0.93 \\
\hline \multicolumn{6}{|l|}{$\begin{array}{l}\text { Economic } \\
\text { status }\end{array}$} \\
\hline Sufficient & $44 / 119$ & 1.0 (reference) & 0.18 & 1.00 (reference) & 0.17 \\
\hline Insufficient & $6 / 31$ & $0.52(0.20-1.34)$ & & $0.48(0.17-1.36)$ & \\
\hline \multicolumn{6}{|l|}{ Type of Family } \\
\hline Single & $22 / 68$ & 1.0 (reference) & 0.98 & 1.00(reference) & 0.88 \\
\hline Joint & $20 / 58$ & $1.07(0.53-2.14)$ & 0.85 & $1.21(0.57-2.56)$ & 0.62 \\
\hline Extended & $8 / 24$ & $1.03(0.40-2.62)$ & 0.95 & $1.16(0.43-3.11)$ & 0.77 \\
\hline
\end{tabular}

*Adjusted for mother's age, education, and income.

Table 6. Associations between Home Related Characteristics and

\section{Children with and without Pneumonia}

\begin{tabular}{|c|c|c|c|c|c|}
\hline \multirow[t]{2}{*}{$\begin{array}{l}\text { Characteris- } \\
\text { tics of Home }\end{array}$} & \multirow{2}{*}{$\begin{array}{l}\text { Pneu- } \\
\text { monia } \\
\text { Yes/No }\end{array}$} & \multicolumn{2}{|l|}{ Unadjusted } & \multicolumn{2}{|c|}{$\begin{array}{l}\text { Adjusted for } \\
\text { Demographics }\end{array}$} \\
\hline & & OR $(95 \% \mathrm{Cl})$ & $P$ & OR $(95 \% \mathrm{Cl})$ & $P$ \\
\hline Ventilation & & & & & 0.77 \\
\hline $\begin{array}{l}\text { Well-venti- } \\
\text { lated }\end{array}$ & $33 / 104$ & 1.00 (reference) & 0.66 & 1.00 (reference) & \\
\hline $\begin{array}{l}\text { Poorly venti- } \\
\text { lated }\end{array}$ & $17 / 46$ & $1.16(0.59-2.30)$ & & $1.11(0.54-2.28)$ & \\
\hline \multicolumn{6}{|l|}{$\begin{array}{l}\text { Smoking } \\
\text { Status of } \\
\text { Parents }\end{array}$} \\
\hline $\begin{array}{l}\text { Neither par- } \\
\text { ent smokes }\end{array}$ & $39 / 129$ & 1.00 (reference) & 0.58 & 1.00 (reference) & 0.48 \\
\hline $\begin{array}{l}\text { Mother } \\
\text { smokes }\end{array}$ & $1 / 3$ & $1.10(0.11-10.90)$ & 0.93 & $1.51(0.14-16.67)$ & 0.73 \\
\hline $\begin{array}{l}\text { Father } \\
\text { smokes }\end{array}$ & $6 / 11$ & $1.80(0.63-5.19)$ & 0.27 & $1.98(0.63-6.25)$ & 0.24 \\
\hline $\begin{array}{l}\text { Both parents } \\
\text { smoke }\end{array}$ & $4 / 7$ & $1.89(0.53-6.80)$ & 0.33 & $2.21(0.56-8.82)$ & 0.26 \\
\hline \multicolumn{6}{|l|}{$\begin{array}{l}\text { Condition of } \\
\text { Chulo }\end{array}$} \\
\hline $\begin{array}{l}\text { Smokeless- } \\
\text { separate }\end{array}$ & $11 / 57$ & 1.00 (reference) & 0.23 & 1.00 (reference) & 0.10 \\
\hline $\begin{array}{l}\text { Smokeless - } \\
\text { same }\end{array}$ & $9 / 22$ & $2.12(0.77-5.81)$ & 0.14 & $2.34(0.80-6.90)$ & 0.12 \\
\hline $\begin{array}{l}\text { Smoke - } \\
\text { separate }\end{array}$ & $20 / 50$ & $2.07(0.91-4.74)$ & 0.08 & $2.76(1.07-7.15)$ & 0.04 \\
\hline Smoke - same & $10 / 21$ & $2.50(0.91-6.65)$ & 0.07 & $3.76(1.20-11.82)$ & 0.02 \\
\hline
\end{tabular}

*Adjusted for mother's age, education, and income.

Table 5 shows that children older than 3 years of age were significantly more likely to present at the pediatric ward with pneumonia than younger children. The association remained significant when adjusted for child's sex. Sex of
Table 5. Associations between Child Related Characteristics and Odds of Children Having Pneumonia

\begin{tabular}{|c|c|c|c|c|c|}
\hline \multirow[t]{2}{*}{$\begin{array}{l}\text { Child } \\
\text { Characteristics }\end{array}$} & \multirow{2}{*}{$\begin{array}{l}\text { Pneu- } \\
\text { monia } \\
\text { Yes/No }\end{array}$} & \multicolumn{2}{|l|}{ Unadjusted } & \multicolumn{2}{|c|}{$\begin{array}{l}\text { Adjusted for } \\
\text { Demographics }\end{array}$} \\
\hline & & OR $(95 \% \mathrm{CI})$ & $P$ & OR $(95 \% \mathrm{Cl})$ & $\mathrm{P}$ \\
\hline \multicolumn{6}{|l|}{ Age of Child } \\
\hline Under 1 year & $14 / 61$ & 1.00 (reference) & -- & 1.00 (reference) & --- \\
\hline $1-3$ years & $23 / 67$ & $1.50(0.71-3.16)$ & 0.29 & $1.51(0.71-3.20)$ & 0.28 \\
\hline Above 3 years & $13 / 22$ & $2.58(1.05-6.32)$ & 0.04 & $2.60(1.06-6.41)$ & 0.04 \\
\hline \multicolumn{6}{|l|}{ Sex of Child } \\
\hline Male & $30 / 81$ & 1.00 (reference) & 0.46 & 1.00 (reference) & 0.43 \\
\hline Female & $20 / 69$ & $0.78(0.41-1.50)$ & & $0.77(0.40-1.48)$ & \\
\hline \multicolumn{6}{|l|}{$\begin{array}{l}\text { Birthplace of } \\
\text { Child }\end{array}$} \\
\hline Home & $14 / 36$ & 1.00 (reference) & 0.57 & 1.00 (reference) & 0.73 \\
\hline $\begin{array}{l}\text { Health Institu- } \\
\text { tion }\end{array}$ & $36 / 114$ & $0.81(0.39-1.67)$ & & $0.87(0.38-1.96)$ & \\
\hline \multicolumn{6}{|l|}{$\begin{array}{l}\text { Birth Weight of } \\
\text { Child }\end{array}$} \\
\hline Normal (GE & $38 / 111$ & 1.00 (reference) & & 1.00 (reference) & \\
\hline $2.5 \mathrm{~kg})$ & & & 0.63 & & 0.41 \\
\hline $\begin{array}{l}\text { Underweight } \\
\text { (LT } 2.5 \mathrm{~kg} \text { ) }\end{array}$ & $12 / 29$ & $0.52(0.20-1.34)$ & & $1.41(0.62-3.22)$ & \\
\hline \multicolumn{6}{|l|}{$\begin{array}{l}\text { Gestational Age } \\
\text { of Child }\end{array}$} \\
\hline$\leq 37$ weeks & $10 / 35$ & 1.00 (reference) & 0.63 & 1.00 (reference) & 0.66 \\
\hline$>37$ weeks & $40 / 115$ & $1.22(0.55-2.68)$ & & $1.20(0.52-2.76)$ & \\
\hline \multicolumn{6}{|l|}{$\begin{array}{l}\text { Colostrum } \\
\text { Feeding }\end{array}$} \\
\hline Yes & $47 / 133$ & 1.00 (reference) & 0.28 & 1.00 (reference) & 0.21 \\
\hline No & $3 / 17$ & $0.50(0.14-1.78)$ & & $0.43(0.12-1.61)$ & \\
\hline \multicolumn{6}{|l|}{$\begin{array}{l}\text { Duration of ex- } \\
\text { clusive Breast } \\
\text { feeding }\end{array}$} \\
\hline 4-6 month & $36 / 103$ & 1.00 (reference) & 0.83 & 1.00 (reference) & 0.87 \\
\hline $\begin{array}{l}\text { Less than } 4 \\
\text { months }\end{array}$ & $9 / 33$ & $0.78(0.34-1.79)$ & 0.56 & $0.79(0.33-1.89)$ & 0.60 \\
\hline $\begin{array}{l}\text { Greater than } 6 \\
\text { months. }\end{array}$ & $5 / 14$ & $1.02(0.34-3.04)$ & 0.97 & $0.97(0.30-3.13)$ & 0.96 \\
\hline \multicolumn{6}{|l|}{$\begin{array}{l}\text { Immunization } \\
\text { Status }\end{array}$} \\
\hline Fully vaccinated & $31 / 86$ & 1.00 (reference) & 0.56 & 1.00 (reference) & 0.42 \\
\hline $\begin{array}{l}\text { Not fully vac- } \\
\text { cinated }\end{array}$ & $19 / 64$ & $0.82(0.43-1.59)$ & & $1.52(0.55-4.22)$ & \\
\hline \multicolumn{6}{|l|}{$\begin{array}{l}\text { Diarrhoea in } \\
\text { past } 3 \text { months }\end{array}$} \\
\hline No & $38 / 82$ & 1.00 (reference) & 0.01 & 1.00 (reference) & 0.01 \\
\hline Yes & $12 / 65$ & $0.40(0.19-0.82)$ & & $0.38(0.18-0.82)$ & \\
\hline \multicolumn{6}{|l|}{$\begin{array}{l}\text { Attending Child } \\
\text { Care Center }\end{array}$} \\
\hline No & $32 / 118$ & 1.00 (reference) & 0.04 & 1.00 (reference) & 0.25 \\
\hline Yes & $18 / 32$ & $2.07(1.03-4.16)$ & & $1.66(0.70-3.97)$ & \\
\hline
\end{tabular}

*Adjusted for child's age and gender

the child was not related to case/control status. Children who were delivered in health institutions had $19 \%$ and $13 \%$ less chance to have pneumonia in both models, but it was statistically insignificant. The children whose birth weight was less than $2.5 \mathrm{~kg}$ had $21 \%$ and $41 \%$ more chance to have pneumonia in unadjusted and adjusted model, respectively; but it was statistically not significant. Similarly gestational age of child at birth and practice of colostrum feeding were not significantly associated with the occurrence of pneumonia. While breast feeding did not appear to be associated with risk of pneumonia, children who were not fully vaccinated had a non- 
significant increased odds of $52 \%$ for having pneumonia than fully vaccinated children in the adjusted model. While attending a child care center was significantly associated with a two-fold increased odds of having pneumonia in the unadjusted model, this association was not significant when the model was adjusted for child's age and sex. The only significant association found among childhood factors was a protective effect of a child having diarrhea in the past three months $(p=.01)$. Children whose mothers reported they had diarrhea in the past three months were $62 \%$ less likely to have presented with pneumonia in the pediatric ward.

Characteristics related to the home that were evaluated included ventilation, parents' smoking habits, and type of chulo used at home (Table 6). In adjusted models, associations with poor ventilation and smoking were not significant. Although not significant, the large odds ratio calculated for both parents smoking is noteworthy as this more than doubled the odds of a child presenting with pneumonia. Significant associations were found for the type of chulo used in the household. Children living in a household with a chulo that produced smoke were at a higher risk of pneumonia than children living in a home using a smokeless chulo in a separate building. The odds of having pneumonia were almost 4 times greater if the chulo was located within the same building (OR: 3.76, 95\% Cl: 1.20-11.82, $\mathrm{p}=0.02$ ).

\section{DISCUSSION}

This study was designed to investigate risk factors for pneumonia presenting at a pediatric ward in a major teaching hospital of Nepal. In general, we found no significant associations between the mother's demographics and risk of her child having pneumonia, although age of the mother approached significance $(p=0.09)$. Women 30 years of age and older were less likely to bring in children with pneumonia than were women age 20-29 who made up the majority of study participants. This may be related to the better awareness of this age group about better outcome of early home treatment for respiratory symptoms preventing the progression to pneumonia needing medical care. In terms of the child, older children (age 3 or more years) were more than twice as likely to have pneumonia than were children less than 1 year. While in unadjusted models, attending a child care center increased the odds of having pneumonia, the association was attenuated when the model was adjusted for child's age and gender. An unexpected finding was that children whose mothers reported their having diarrhea in the past few 3 months were less likely to present with pneumonia. A possible explanation for this may be that these children were perhaps treated with zinc or antibiotics that simultaneously retained a protective effect against pneumonia, similar to the findings of Lassi ZS, Haider BA,
Bhutta ZA. ${ }^{6}$ In terms of the family household, a greater than two-fold odds of having pneumonia was found when both parents smoked, although this association was not significant and may reflect the small number of smokers we had in our sample. An important finding of this study was the increased risk of pneumonia found in children whose homes used a smoky chulo. The odds were almost threefold if the chulo was in a separate building and almost fourfold when it was in the same building as the main house. Both of these associations were significant. This finding is supported by other studies investigating the home environment. ${ }^{10,11,14,18,19}$ The present study did not show relationships with religion, education status of mothers,or economic status of family on the occurrence of pneumonia in children. While these findings are similar to one study, ${ }^{7}$ many others have found low education and other measures of low socioeconomic status to be associated with risk of pneumonia in children. ${ }^{8-11}$ We believe that small sample size, misclassification, or other methodological issues may explain the differences found among studies.

This study found no associations between place of child's delivery or joint/extended families living in one household to be related to pneumonia status. The study done by MR Savitha found overcrowding significantly associated with acute respiratory illness (ARI). ${ }^{11}$ In our study, low birth weight, partial immunization and partially breast fed showed insignificant but increased odds of getting pneumonia. While similarly to us, a study reported no associations, ${ }^{9}$ most studies have reported an increased risk of these factors. ${ }^{10,12-14}$ These differences, once again, may be due to small sample size or other issues related to methodology of the negative studies. The lack of association between day care attendance and pneumonia in the adjusted model and pneumonia may be attributed to improvement of day care center now days. This report supports the study by Darkwa AG and Igor Rudan, Cynthia Boschi-Pinto but is in contrast to the results of FA Ujunwa and CT Ezeonu. . $^{94,15}$

The finding that children who had diarrhea in the past 3 months were $60 \%$ less likely to present with pneumonia warrants additional discussion. This contrasts with results of studies in Kenya and other low resource settings where co-morbid diarrhea was correlated with increased rates of pneumonia. ${ }^{7,14,16}$ Although we acknowledge that children with concomitant illness may have their immunity lowered making them more susceptible to severe disease, our results could have been due to treatment for the diarrhea. Since the mothers had access to good health care at Dhulikhel Hospital, it is very possible that antibiotics and zinc were provided which would have protected them from subsequent pneumonia. Another reason that this result occurred may be due to the design of the study and selection of controls. As diarrhea remains a common child illness in Nepal, more children with diarrhea but not pneumonia may have been matched to cases producing results found here. 
The presence of smoke in the home as a risk factor for respiratory illnesses in children was reinforced in this study. Although not significant, children's exposure to parents' smoking showed about a two-fold increased odds when fathers or both parents smoked, considered a large risk in epidemiologic studies. Statistical significance may have been limited due to low numbers of smokers in this study or the relatively greater time spent by children outside the home. The present study also showed that children living in homes with a smoky chulo in the same building had an almost four-fold significant increased odds of pneumonia. This finding was supported by studies conducted by Dherani M, Pope D. et al. ${ }^{18}$, Bruce N, Weber M, Arana B, et al ${ }^{19}$; M.R. Savitha ${ }^{11}$; IgorRudan, Cynthia Boschi-Pinto, ${ }^{14}$ and Shah N, Ramankutty V. ${ }^{10}$ Provision of clean fuels, householder's education about effects of smoke on children, and modification of stoves are potential measures to decrease this risk. But, as the present study was hospital-based,these finding may not be representative of all pneumonia cases in the community. These results would benefit from additional population-based research.

\section{REFERENCES}

1. Black RE, Morris SS, Bryce J. Where and why are 10 million children dying every year?. Lancet. 2003; 361(9376):2226-2234.

2. WHO/ UNICEF, Report on ARI. CDD/ARI section, Child health division, department of health service, Ministry of Health, Nepal (1997)

3. MI Bari, AB Siddiqui, T Alam, A Hossain. Risk Factors of Pneumonia in Children-A Community Survey: 2007; doi: 10.3329/taj.v20i2.3072 TAJ 2007; 20(2): 122-126

4. New-Era Project, Ministry of Health. Nepal Demographic and Health Survey (2006).

5. Banstola A. The Epidemiology of hospitalization for pneumonia in children under 5 in the rural western region of Nepal: A Descriptive study. PLos ONE 8 (8): 2013; e71311.doi:10.1371/journal. pone.0071311.

6. Lassi ZS, Haider BA, BhuttaZA. Zinc supplementation for the prevention of pneumonia in children aged two to 59 months. Published Online: 8 DEC 2010 DOI: 10.1002/14651858.CD005978. pub2.

7. Dickens Onyango, Gideon Kikuvi, Evans Amukoye, Jared Omolo. Risk factors of severe pneumonia among children aged 2-59 months in western Kenya: a case control study. The Pan African Medical Journal. 2012;13:45

8. Ballard TJ, Neumann CG. The effects of malnutrition, parental literacy and household crowding on acute lower respiratory infections in young Kenyan children. J Trop Pediatr 1995;41:8-13. Abstract/FREE Full Text

9. Darkwa, A. G. Risk Factors for Pneumonia in Children Under Five at Komfo Anokye Teaching Hospital, 2012-07, http://hdl.handle. net/123456789/5493, retrived on $15 / 2 / 2015$

10. Shah N, Ramankutty V, Premila PG, Sathy N. Risk factors for severe pneumonia in children in south Kerala: a hospital-based case-control study. J Trop Pediatr. 1994 Aug;40(4):201-6. from http://www.ncbi. nlm.nih.gov/pubmed/7932932 retrived on 11/08/2014.

\section{CONCLUSION}

As acute respiratory illnesses (ARI) are preventable, family members and trained specialists can play significant roles to reduce their occurrence through health promotion, preventive activities and effective child health programs5. In this study, our most important finding involved the home environment, specifically the presence of smoke in the home due to use of a smoky chulo or (suggested) smoking by parents - both modifiable risk factors. Having endorsed the Millennium Declaration, the Government of Nepal is committed to the millennium development goals (MDGs) which are targeting under 5 mortality. Reliable and comparable analysis of risks to respiratory health along with programs to educate parents in prevention of ARI is essential for reaching these goals.

\section{ACKNOWLEDGEMENT}

The authors would like to thank Khagendra Acharya (Assistant Professor. K.U.) for English language editing and all participants of this study for their valuable help and enthusiasm in making this study possible.

11. M.R. Savitha, S.B Nandeeshwara, M.J. Pradeep Kumar, Farhanul- haque and C.K. Raju: Modifiable Risk Factors for Acute Lower Respiratory Tract Infection. Indian Journal of Paediatrics 2007 May; 74:.

12. Nafstad P, Jaakkola JJ, Hagen JA, Botten G, Kongerud J. Breastfeeding, maternal smoking and lower respiratory tract infections. Eur Respir J 1996;9:2623-9.

13. Chantry $\mathrm{CJ}$, Howard CR, Auinger P. Full breastfeeding duration and associated decrease in respiratory tract infection in US children. Pediatrics 2006;117:425-32.

14. IgorRudan, Cynthia Boschi-Pinto, ZrinkaBiloglav Kim Mulholland Harr Campbell Epidemiology and etiology of Childhood pneumonia, Bull World Health Organ. 2008 May; 86(5): 408-416.

15. FA Ujunwa and CT Ezeonu, Risk Factors for Acute Lower Respiratory Tract Infections in Under-five Children in Enugu Southeast Nigeria, Ann Med Health Sci Res. 2014 Jan-Feb; 4(1): 95-99.

16. Moustaki M, Nicolaidou P, Stefos E, Vlachou V, Patsouri P, Fretzayas A. Is there an association between wheezing and pneumonia? Allergollmmunopathol (Madr) 2010;38:4-Cross Ref Medline Search Google Scholar-14

17. S. Broor, R.M. Pandey, M. Ghosh, R.S. Maitreyi, Rakesh Loudha, TanuSigghal et al. Risk factors for Severe Acute Lower Respiratory Tract Infection in Under Five Children. Indian Pediatrics 2001; 38:1361-1369

18. Dherani M, Pope D, Mascarenhas M, Smith KR, Weber M, Bruce N. Indoor air pollution from unprocessed solid fuel use and pneumonia risk in children aged under five years: a systematic review and metaanalysis. Bull World Health Organ 2008;86:390-8C.Cross Ref Medline Web of Science Search Google Scholar.

19. Bruce N, Weber M, Arana B, et al Pneumonia case-finding in the RESPIRE Guatemala indoor air pollution trial: standardizing methods for resource-poor settings. Bull World Health Organ 2007;85:535-44. Cross Ref Medline Web of Science Search Google Scholar 